\title{
Effect of Intensive Care Provided by Nurse Practitioners for Postoperative Patients: A Retrospective Observational Before-and-after Study
}

Kazunao Mori ( $\sim$ kazu21.np@gmail.com )

Division of Nursing, Aichi Medical University Hospital https://orcid.org/0000-0003-2986-8033

\section{Satoshi Makino}

Aichi Medical University Hospital

Takuya Takabayashi

Aichi Medical University Hospital

Masahiro Kurosawa

Aichi Medical University

Wataru Ohashi

Aichi Medical University Hospital

Yoko Tsukamoto

Health Sciences University of Hokkaido

Masatoshi Okumura

Aichi Medical University

Yoshihito Fujita

Aichi Medical University

Yoshihiro Fujiwara

Aichi Medical University

\section{Research}

Keywords: nurse practitioners, intensive care, ventilation, hospital

Posted Date: August 12th, 2020

DOl: https://doi.org/10.21203/rs.3.rs-52607/v1

License: (c) (i) This work is licensed under a Creative Commons Attribution 4.0 International License.

Read Full License 


\section{Effect of intensive care provided by nurse practitioners for}

postoperative patients: a retrospective observational before-and-after study

Kazunao Mori ${ }^{1 *}$, Satoshi Makino ${ }^{1}$, Takuya Takabayashi ${ }^{1}$, Masahiro Kurosawa ${ }^{2}$, Wataru Ohashi $^{3}$, Yoko Tsukamoto ${ }^{4}$, Masatoshi Okumura ${ }^{5}$, Yoshihito Fujita ${ }^{5}$ and Yoshihiro Fujiwara $^{5}$

${ }^{1}$ Division of Nursing, Aichi Medical University Hospital

${ }^{2}$ Department of Nursing, Aichi Medical University

${ }^{3}$ Division of Biostatistics, Clinical Research Center, Aichi Medical University Hospital

${ }^{4}$ Department of Nursing and Social Sciences, Health Sciences University of Hokkaido

${ }^{5}$ Department of Anesthesiology, Aichi Medical University

*Corresponding author: Kazunao Mori, Division of Nursing, Aichi Medical University Hospital, 1-1 Yazakokarimata, Nagakute, Aichi 480-1195, Japan

Tel: +81-561-62-3311 (Ext. 78324)

E-mail: kazu21.np@gmail.com 


\section{Abstract}

Background: Since April 2016, nurse practitioners (NPs) have provided interprofessional team collaborative care for postoperative patients in our intensive care unit (ICU). To assess the effectiveness of NP practice, we compared patient outcomes with and without NP care in our ICU.

Methods: This retrospective observational cohort study was undertaken at Aichi Medical University Hospital in Japan from 01 April 2015 and 31 March 2017. Mechanicallyventilated postoperative patients who stayed in the ICU for $>2$ days were eligible for inclusion. We compared the patient outcomes between the period without NP care from April 2015 to March 2016 (physician group) and the period with NP care from April 2016 to May 2017 (NP-physician group). The primary outcome was ICU length of stay. Secondary outcomes were mechanical ventilation days and total hospital length of stay. To further determine the factors associated with ICU length of stay, multiple regression analysis was performed for age, Acute Physiology and Chronic Health Evaluation (APACHE) II score, NP-physician group, and physician group.

Results: A total of 387 patients were included in the study. Among these patients, 213 were assigned to the NP-physician group and 174 were assigned to the physician group. The NP-physician group had shorter ICU length of stay than the physician group $(4.8 \pm$ 4.8 days versus $6.7 \pm 10.3$ days, $p<0.021$ ). There were no significant differences between the two groups in mechanical ventilation days or total hospital length of stay. In the multiple regression analysis for ICU length of stay, APACHE II score and NP-physician group were associated with ICU length of stay. Specifically, NP-physician group reduced ICU length of stay by 2.6 days $(p=0.003)$, and APACHE II score one-point decrease reduced ICU length of stay by 0.2 days $(p=0.012)$. 
Conclusions: The present study demonstrated shorter ICU length of stay in patients with NP care and a significant association between NP participation in the ICU team and ICU length of stay.

Keywords: nurse practitioner, ICU length of stay, postoperative patient, intensive care unit 


\section{Background}

The world's population continues to grow older in most countries as people tend to live longer. In 2015 , people aged $\geq 65$ years reached $8.5 \%$ of the total population [1]. In 2050 , people aged $\geq 65$ years will comprise $28 \%$ of the general population in Japan $[1,2]$.

Recent advances in anesthesia and surgical techniques have enabled surgery for elderly people. The median age of the entire intensive care unit (ICU) population is already $>65$ years in many countries $[3,4]$. However, elderly patients experience more postoperative complications, such as pulmonary events, cardiac issues, venous thrombosis, delirium, and postoperative cognitive dysfunction $[5,6]$. Therefore, these patients have complex care requirements and concurrent comorbidities that compound their clinical care management needs.

In the United States, there are 270,000 certified nurse practitioners (NPs). Data for models of care that integrate NPs in acute and critical care settings and the outcomes of this care are essential to meet the needs of acute and critically ill patients [7]. NPs have expanded in ICU settings since 1990 and reduced the ICU length of stay and mortality [8]. Meanwhile, Japan initiated NP education in 2010, and NP care was adopted in Aichi Medical University Hospital in 2015.

NPs provide inter-professional team collaborative care for postoperative patients with multiple medical problems in the ICU setting. NPs have an important role in interprofessional team medicine, because they enhance communication by seeking consultations and facilitate bridging across disciplines. NPs spend more time on clinical practice and consultation. Although the role of NPs is recognized in many countries, no data exist on the specific numbers of NPs practicing in Japan. To assess the effectiveness of NP practice, we compared outcomes for postoperative patients in the ICU who received 
NP-physician care and physician-only care.

This study involved an investigation of the practice of NP participation in the ICU teams at Aichi Medical University Hospital. As a background, NPs have a similar role to physicians in providing care to critically ill patients. Physicians and NPs both provide multidisciplinary management for patients together with nurses, physiotherapists, and pharmacists. However, the roles of physicians and NPs differ in a few fundamental aspects. For example, NPs can undertake tasks such as intravenous route, arterial line, and intubation with physicians, administration of drugs, and changes to ventilator settings. However, NPs cannot undertake activities such as prescription of narcotics and administration of a central venous route or nerve blockade.

NPs share information on treatment plans with nurses, pharmacists, and physiotherapists, and consult physicians about patient understanding in the treatment environment from the nurses' perspective. NPs must work with physicians, nurses, pharmacists, and physiotherapists to assess the patient condition and intervene to prevent deterioration of the condition. However, there tends to be insufficient inter-professional communication, because physicians, nurses, physiotherapists, and pharmacists rarely discuss treatment plans. For that reason, NPs need to promote inter-professional communication.

NPs provide care before and after extubation and act to prevent complications such as re-intubation, ventilator-associated events, ICU-acquired weakness, delirium, and postintensive care syndrome. In addition, they manage fluctuations in breathing and blood pressure during mobilization. NPs enhance effectiveness of care through a collaborative approach to patients. That approach means building a good relationship between NPs and patients. NPs provide effective communication to build a therapeutic relationship with 
patients and their families, and promote patient and family participation in the development and revision of the plan of care. NPs evaluate rapidly changing patient conditions and determine the necessity for medical treatment. NPs are trained to consult physicians if physician treatment is needed and to respond when no physician treatment is needed. In this way, NPs can provide both therapeutic interventions and nursing interventions. The aim of this study was to compare patient outcomes with and without NP care in the ICU to assess the effectiveness of NP practice.

\section{Materials and methods}

\section{Study design}

This was a retrospective before-and-after study from 01 April 2015 and 31 March 2017. From April 2015 to March 2016, there was no NPs in the ICU team (physician group). From April 2016 to March of 2017, there were two NPs and one physician in the ICU team (NP-physician group). The NPs worked 8 hours during day time or 16 hours during night time.

\section{Study population}

Mechanically-ventilated postoperative patients who stayed in the ICU for $>2$ days were eligible for inclusion. The exclusion criteria were two or more admissions to the ICU and no detailed descriptions in the medical records.

\section{Study outcomes}

The primary outcome was ICU length of stay. The secondary outcomes were mechanical ventilation days and total hospital length of stay. To further determine whether these 
factors were associated with NP care, we investigated the impacts of these factors on the outcomes.

\section{Statistical analysis}

Descriptive data are presented as number and percentage and continuous data are described as mean \pm standard deviation. Categorical variables were compared using Student's $t$-test. A multiple regression analysis was carried out to evaluate the relationships between ICU length of stay, age, Acute Physiology and Chronic Health Evaluation (APACHE) II score [9], and the two groups. Differences with $p$-values of $<0.05$ were considered statistically significant. SPSS version 23.0 software (SPSS Inc., Chicago, IL) was used for all statistical analyses.

\section{Ethical considerations}

This study was approved by the Ethical Committee of Aichi Medical University, Japan (approval number: 2018-H287). The collected data were guaranteed anonymity and confidentiality during the study period. Informed consent was waived by the Institutional Review Board because of the retrospective design of the study.

\section{Results}

\section{Demographic characteristics}

During the 2-year study period, 410 postoperative patients with mechanical ventilator assistance were admitted to the ICU. A total of 23 patients were excluded, comprising 12 patients with no detailed descriptions in their medical records and 11 patients with two or more admissions to the ICU. After exclusion of these patients, 387 participants were 
enrolled in the study (Fig. 1).

The baseline characteristics of the patients are summarized in Table 1. Mean age was $65.6 \pm 14.1$ years in the physician group and $64.0 \pm 14.8$ years in the NP-physician group. Baseline APACHE II score $(28.6 \pm 5.5$ versus $32.0 \pm 4.8, p<0.001)$ differed significantly between the two groups. There were no significant differences between the two groups in age, body mass index, operation time, or anesthesia time.

\section{Impact of NP participation in the ICU team}

Regarding the clinical outcomes, the NP-physician group had shorter ICU length of stay than the physician group $(4.8 \pm 4.8$ days versus $6.7 \pm 10.3$ days, $p<0.021)$. There were no significant differences between the two groups in mechanical ventilation days or total hospital length of stay (Table 2). Age and disease severity of ICU patients are known factors associated with ICU length of stay. Therefore, we added these factors to a multiple regression model to determine the factors for ICU length of stay. The results of the multiple regression analysis are presented in Table 3 . In the model, age had no impact on ICU length of stay. However, APACHE II score and NP-physician group were associated with ICU length of stay. Specifically, APACHE II score one-point decrease reduced ICU length of stay by 0.2 days and NP-physician group reduced ICU length of stay by 2.6 days.

\section{Discussion}

In this study, we found shorter ICU length of stay in the NP-physician group and a significant association between NP participation in the ICU team and ICU length of stay. Although APACHE II score was higher in the NP-physician group compared with the 
physician group, ICU length of stay was reduced by 2.6 days in the NP-physician group. Several studies have reported that NP care and physician care showed no significant differences in hospital length of stay or mortality [10,11], and it has become increasingly clear that the effect of NP care in the ICU setting is important. The present results suggest that NPs have a different role than physicians. We consider that three different reasons underlie the good results in the present study.

The first reason is that NPs are key people in an inter-professional team. NPs can collaborate with nurses and other professionals [12]. NPs also act to prevent complications in critically ill patients. Even when nurses have difficulty in assessing the patient condition, NPs can assess complex health problems using pathophysiology and diagnostics. NP also provide direct care with supervisors when needed [13]. In such cases, NPs use the concept of "knotworking" in hospitals [10]. Rapid knotworking can solve problems in rapidly-changing patient conditions. Brief knotworking is used for coordination and integration of inter-professional team collaborative care. NPs employ knotworking within a process to solve problems, enable timely care provision, and decrease errors. NPs promote team medical care, and practice knotworking in interprofessional teams [10]. In fact, NPs spend time in clinical practice and consultation within hospitals, and receive more consultation than nurses as well as consultation with paramedics and residents [14].

The second reason is that the styles of NP communication may have impacted patient outcomes. Communication is an important factor in care for critically ill patients, because the patient condition must be shared among different professionals. Communication is necessary for inter-professional collaboration. In a previous study, physicians felt that they were collaborating with nurses, but nurses felt that they were communicating more 
with NPs than with physicians [15]. This shows the possibility of a good relationship between NPs and nurses. Meanwhile, NPs provide biomedical and biopsychosocial communication with patients, especially biopsychosocial communication. The biopsychosocial style of communication actively engages patients in discussions and decision-making processes. This style of communication is also referred to as patientcentered communication. Incorporation of patient-centered communication into NP practice is associated with improvement of patient outcomes [16]. The present results suggest that the NP-patient relationship may also be associated with shorter ICU length of stay.

The third reason is that NPs provide a continuum of care, practicing care according to the patient sleep cycle and delirium care at night. When patients complain of insomnia, NPs can investigate the cause of the insomnia and prepare an environment in which the patients can sleep. In delirium care, NPs assess the nature of the complaint and provide interventions. Therefore, NPs provide care tailored toward patient needs. Even at night, NPs are required to evaluate patients, support nurses, provide direct care, and consult with nurses [17].

This study had four limitations. First, it was a single-institution study at a university hospital. Therefore, the results may not be generalizable to other hospitals. Second, it was a retrospective review, rather than a prospective randomized controlled trial. In fact, it may not have been possible to carry out a randomized controlled trial, because we were unable to divide the patients into groups with or without NP care in our setting. The numbers of patients could not be unified in the two groups. Third, the study subjects were postoperative patients with mechanical ventilator use. Therefore, the results may not be indicated in emergency and medical ICU settings. Finally, we did not investigate the 
relationships between implementation of NPs in the ICU and incidence of complications or mortality. In the future, it is necessary to investigate patient outcomes such as mortality before and after NP intervention in Japan.

\section{Conclusions}

We found shorter ICU length of stay in the NP-physician group and a significant association between NP participation in the ICU team and ICU length of stay. Our study provides evidence that NPs provide effective care for postoperative patients with mechanical ventilation in the ICU. This is the result of direct care for patients while activating collaboration between doctors and nurses or multi-disciplinary types.

\section{List of abbreviations}

APACHE: acute physiology and chronic health evaluation; NP: nurse practitioner.

\section{Declarations}

\section{Ethics approval and consent to participate}

Our institutional ethics review board approved this study. The review board waived the need for written informed consent from individual patients.

\section{Consent for publication}

Not applicable. 


\section{Availability of data and materials}

All data generated or analyzed during this study are included in this published article.

\section{Competing interests}

The authors declare that they have no competing interests.

\section{Funding}

None.

\section{Authors' contributions}

KM and YT conceptualized the study. KM, SM, and TT performed data curation. KM, WO, and YT carried out the formal analysis. KM and YT performed the investigation. YT designed the methodology. KM administered the project. MO, YFujita, and YFujiwara supervised the study.

\section{Acknowledgments}

The authors thank Alison Sherwin, PhD, from Edanz Group (https://en-authorservices.edanzgroup.com/ac) for editing a draft of this manuscript. 


\section{References}

1. He W, Goodkind D, Kowal P. An Aging World: 2015. US Census Bureau, 2016. Available

from: https://www.census.gov/content/dam/Census/library/publications/2016/demo/p9516-1.pdf.

2. Janssen TL, Steyerberg EW, Faes MC, Wijsman JH, Gobardhan PD, Ho GH, van der Laan L. Risk factors for postoperative delirium after elective major abdominal surgery in elderly patients: a cohort study. Int J Surg 2019;71:29-35.

3. Vallet H, Moïsi L, Thomas C, Guidet B, Boumendil A. Acute critically ill elderly patients: what about long term caregiver burden? J Crit Care 2019;54:180-4.

4. Flaatten H, de Lange DW, Artigas A, Bin D, Moreno R, Christensen S, Joynt GM, Bagshaw SM, Sprung CL, Benoit D, Soares M, Guidet B. The status of intensive care medicine research and a future agenda for very old patients in the ICU. Intensive Care Med 2017;43:1319-28.

5. Angarita FA, Acuna SA, Cordeiro E, Elnahas A, Sutradhar S, Jackson T, Cil TD. Thirty-day postoperative morbidity and mortality in elderly women with breast cancer: an analysis of the NSQIP database. Breast Cancer Res Treat 2018;170:3739.

6. Bekker AY, Weeks EJ. Cognitive function after anaesthesia in the elderly. Best Pract Res Clin Anaesthesiol 2003;17:259-72.

7. Kleinpell RM, Grabenkort WR, Kapu AN, Constantine R, Sicoutris C. Nurse practitioners and physician assistants in acute and critical care: a concise review of 
the literature and data 2008-2018. Crit Care Med 2019;47:1442-9.

8. Landsperger JS, Semler MW, Wang L, Byrne DW, Wheeler AP. Outcomes of nurse practitioner-delivered critical care: a prospective cohort study. Chest 2016;149:114654.

9. Knaus WA, Draper EA, Wagner DP, Zimmerman JE. APACHE II: a severity of disease classification system. Crit Care Med 1985;13:818-29.

10. Hurlock-Chorostecki C, van Soeren M, MacMillan K, Sidani S, Donald F, Reeves S. Nurse practitioner interactions in acute and long-term care: an exploration of the role of knotworking in supporting interprofessional collaboration. BMC Nurs 2015;14:50.

11. Gershengorn HB, Wunsch H, Wahab R, Leaf D, Brodie D, Li G, Factor P. Impact of nonphysician staffing on outcomes in a medical ICU. Chest 2011;139:1347-53.

12. Hurlock-Chorostecki C, Forchuk C, Orchard C, van Soeren M, Reeves S. Labour saver or building a cohesive interprofessional team? The role of the nurse practitioner within hospitals. J Interprof Care 2014;28:260-6.

13. American Association of College of Nursing. Adult-Gerontology Acute Care Nurse Practitioner Competencies 2012. 2012. Available from: https://cdn.ymaws.com/www.nonpf.org/resource/resmgr/competencies/adultgeroaccompsfinal2012.pdf

14. Van Soeren M, Hurlock-Chorostecki C, Reeves S. The role of nurse practitioners in hospital settings: implications for interprofessional practice. J Interprof Care $2011 ; 25: 245-51$

15. Vazirani S, Hays RD, Shapiro MF, Cowan M. Effect of a multidisciplinary intervention on communication and collaboration among physicians and nurses. Am J Crit Care 2005;14:71-7. 
16. Charlton CR, Dearing KS, Berry JA, Johnson MJ. Nurse practitioners' communication styles and their impact on patient outcomes: an integrated literature review. J Am Acad Nurse Pract 2008;20:382-8.

17. Clark S, Paul F. The role of the nurse practitioner within the hospital at night service. Br J Nurs 2012;21:1132-7. 


\section{Figure Legend}

Figure 1 Derivation of the study cohort.

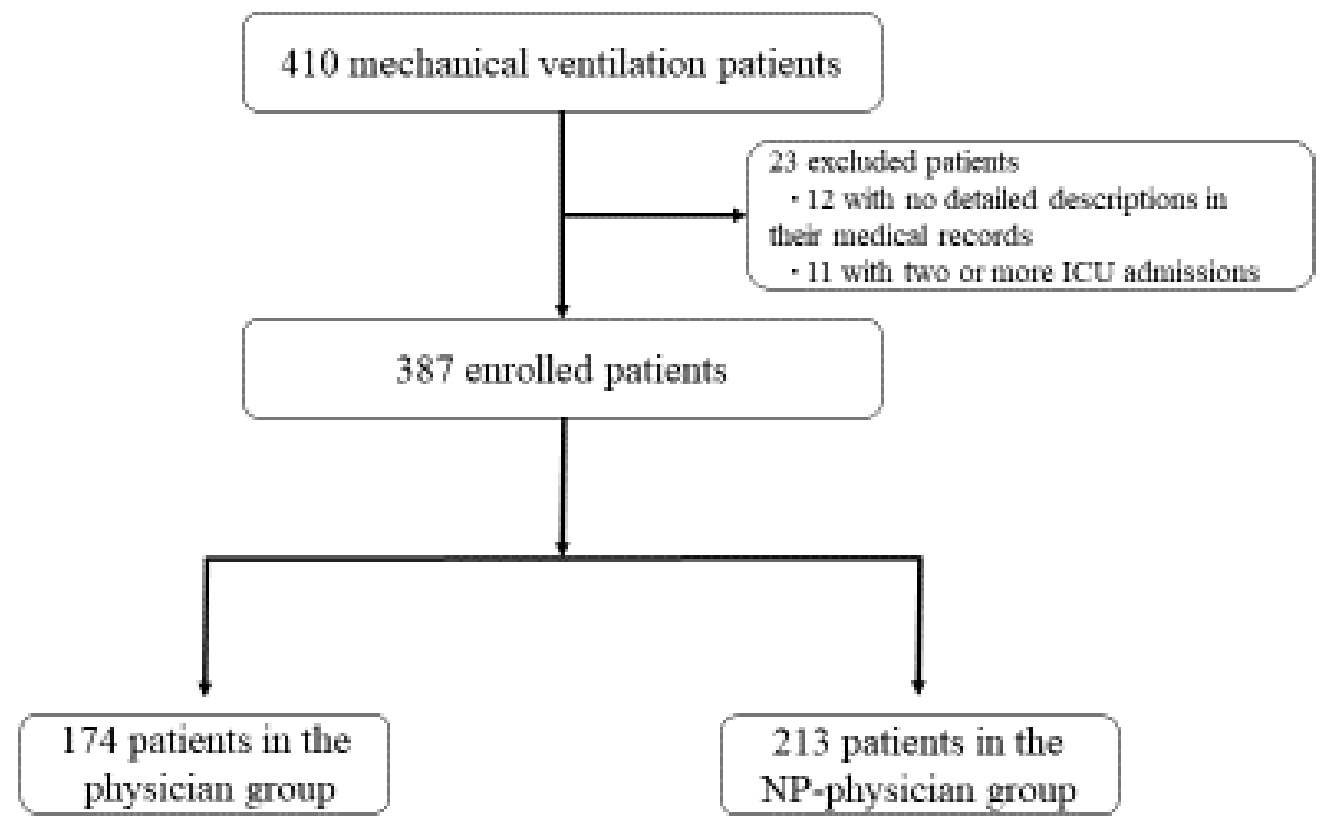

Figure 1 
Table 1 Demographic characteristics of the study patients in the ICU

\begin{tabular}{lccc}
\hline Characteristic & $\begin{array}{c}\text { Physician } \\
\text { group } \\
(n=174)\end{array}$ & $\begin{array}{c}\text { NP-physician } \\
\text { group } \\
(n=213)\end{array}$ & $p$-value \\
\hline Age (yr), mean (SD) & $65.6 \pm 14.1$ & $64.0 \pm 14.8$ & 0.288 \\
BMI, mean (SD) & $22.2 \pm 4.1$ & $21.2 \pm 3.6$ & 0.013 \\
APACHE II score, mean (SD) & $28.6 \pm 5.5$ & $32.0 \pm 4.8$ & $<0.001$ \\
$\quad$ Missing, $n(\%)$ & & $1(0.005)$ & 0.243 \\
Operation time (min), mean(SD) & $409.6 \pm 191.6$ & $387.1 \pm 185.4$ & $1(0.005)$ \\
$\quad$ Missing, $n$ (\%) & $506.3 \pm 199.0$ & $514.0 \pm 812.0$ & 0.903 \\
Anesthesia time (min), mean (SD) & & & \\
\hline
\end{tabular}


Table 2 Patient outcomes

\begin{tabular}{lccc}
\hline Patient outcomes & $\begin{array}{c}\text { Physician } \\
\text { group } \\
(\eta=174)\end{array}$ & $\begin{array}{c}\text { NP-physician } \\
\text { group } \\
(j=213)\end{array}$ & $p$-value \\
\hline $\begin{array}{l}\text { Mechanical ventilation days, mean (SD) } \\
\text { Missing, } n(\%)\end{array}$ & $3.2 \pm 6.0$ & $2.2 \pm 6.8$ & 0.243 \\
ICU length of stay, mean (SD) & $6.7 \pm 10.3$ & $3(0.01)$ & 0.021 \\
$\quad$ Missing, $n(\%)$ & & $4.8 \pm 4.8$ & $2(0.009)$ \\
Total hospital length of stay (days), mean (SD) & $40.5 \pm 58.2$ & $37.1 \pm 36.6$ & 0.483 \\
$\quad$ Missing, $n(\%)$ & & $1(0.005)$ & \\
\hline
\end{tabular}


Table 3 Multiple regression analysis for ICU length of stay

\begin{tabular}{lccccc}
\hline Variables & B & SE & $\beta$ & T-value & $p$-value \\
\hline Age & -0.041 & 0.029 & -0.073 & -1.400 & 0.162 \\
APACHE II score & 0.203 & 0.080 & 0.140 & 2.538 & 0.012 \\
NP-physician group & -2.562 & 0.845 & -0.162 & -3.031 & 0.003 \\
\hline
\end{tabular}


Figures

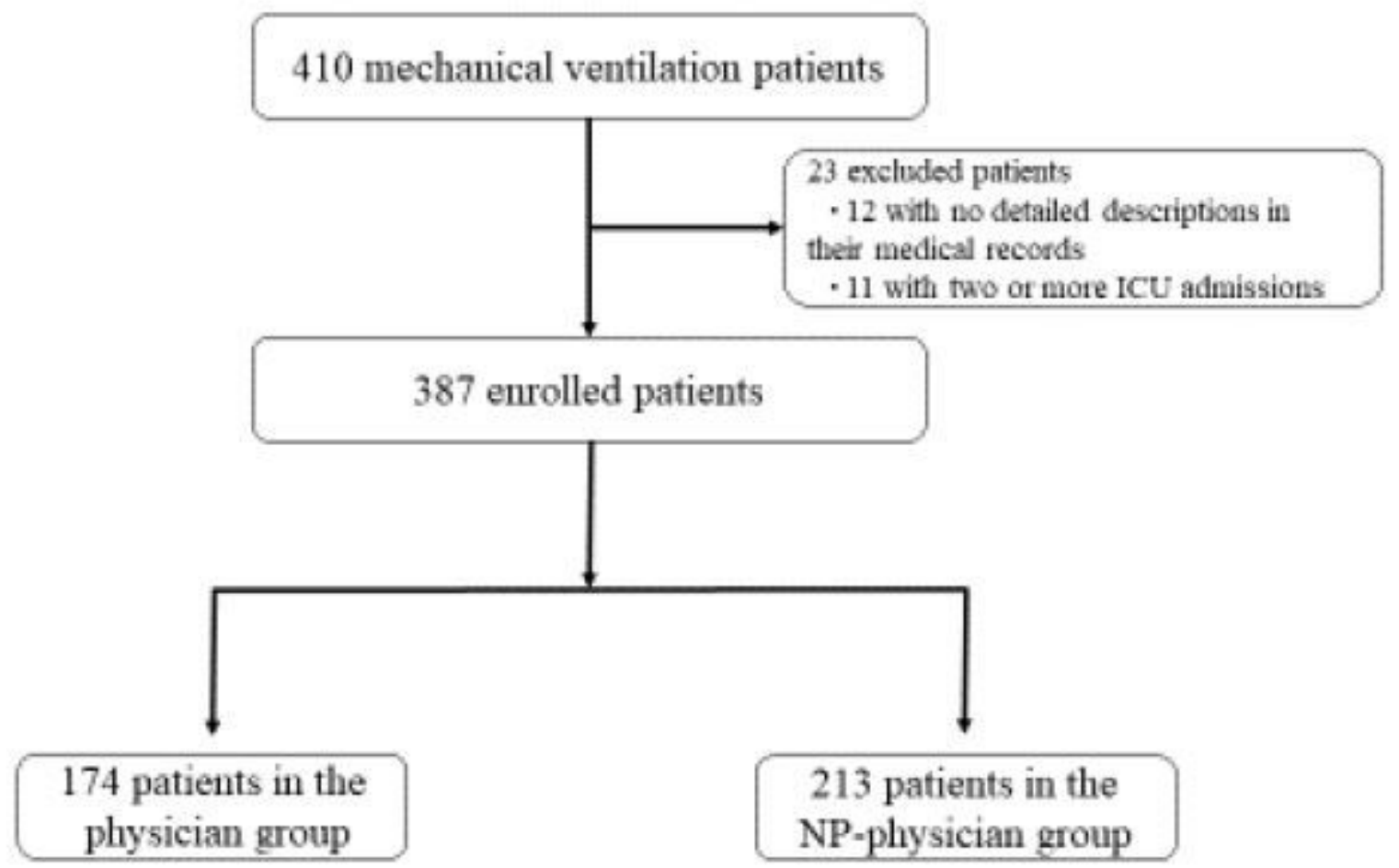

Figure 1

Figure 1

Derivation of the study cohort. 